\title{
The case for virtual register machines ${ }^{\text {花 }}$
}

\author{
David Gregg ${ }^{\mathrm{a}, *}$, Andrew Beatty ${ }^{\mathrm{a}}, \mathrm{Kevin}$ Casey $^{\mathrm{a}}$, Brian Davis ${ }^{\mathrm{a}}$, \\ Andy Nisbet ${ }^{\mathrm{b}}$ \\ ${ }^{a}$ Department of Computer Science, Trinity College Dublin, Dublin 2, Ireland \\ ${ }^{\mathrm{b}}$ Department of Computing and Mathematics, Manchester Metropolitan University, Manchester M15GD, UK
}

Received 10 December 2003; received in revised form 8 July 2004; accepted 9 August 2004

Available online 12 May 2005

\begin{abstract}
Virtual machines (VMs) are a popular target for language implementers. A long-running question in the design of virtual machines has been whether stack or register architectures can be implemented more efficiently with an interpreter. Many designers favour stack architectures since the location of operands is implicit in the stack pointer. In contrast, the operands of register machine instructions must be specified explicitly. In this paper, we present a working system for translating stack-based Java virtual machine (JVM) code to a simple register code. We describe the translation process, the complicated parts of the JVM which make translation more difficult, and the optimisations needed to eliminate copy instructions. Experimental results show that a register format reduced the number of executed instructions by $34.88 \%$, while increasing the number of bytecode loads by an average of $44.81 \%$. Overall, this corresponds to an increase of 2.32 loads for each dispatch removed. We believe that the high cost of dispatches makes register machines attractive even at the cost of increased loads.
\end{abstract}

(C) 2005 Elsevier B.V. All rights reserved.

Keywords: Interpreter; Virtual machine; Register architecture; Stack architecture

\footnotetext{
This work was supported by Enterprise Ireland Research Innovation Fund, Grant IF/2001/350.

* Corresponding author.

E-mail addresses: David.Gregg@cs.tcd.ie (D. Gregg), a.nisbet@mmu.ac.uk (A. Nisbet).
} 


\section{Motivation}

Virtual machines (VMs) are a popular target for language implementers who wish to distribute programs in a portable, architecture-neutral format, which can easily be interpreted or compiled. Virtual machine code is also a standard intermediate format for efficient, general-purpose virtual machine interpreters. The most popular virtual machines use a virtual stack architecture for evaluating expressions, rather than the register architectures that are commonly used in real processors.

A long-running question in the design of virtual machines has been whether stack or register architectures can be implemented more efficiently with an interpreter. Many designers favour stack architectures since the location of operands is implicit in the stack pointer $[5,6,19]$. In contrast, the operands of register machine instructions must be specified explicitly. The interpreter must fetch these operands from the virtual machine code, increasing the interpreter overhead, when compared with a stack architecture. It is also widely believed that stack architectures allow more compact virtual machine code, again because operands are specified implicitly. In addition, stack code is easier to generate in the compiler than register code. At the very least, a stack machine eliminates the need for a complicated register allocator.

For these reasons, a stack machine was chosen as the intermediate representation for the original implementation of Pascal. The Pascal source code was compiled to P-code, which ran on a virtual machine, the most popular implementation of which was the P4 [24]. P-code was the first really successful virtual machine, and it helped establish the concept as a real alternative for language implementations. Later, a stack architecture was also chosen for the virtual machine in the ground-breaking Smalltalk programming environment $[16,20]$. Since then, stack architectures have been used as the intermediate representations for several popular virtual machines including the Java VM and .NET VM.

More recently, a number of authors and implementors of virtual machines have suggested that virtual register machines could be more efficient. Gregg et al. [13] mentioned the possibility in a general discussion of interpreter optimisations. Furthermore, the Parrot VM - the intermediate representation for Perl 6 - will use a register architecture because the implementers belief in the superiority of register machines. The Parrot VM has provoked a number of lively debates on newsgroups such as comp.compilers and comp.lang.perl on the relative merits of virtual stack and register machines. Despite the controversy, neither side has presented significant quantitative results comparing the two approaches, so no conclusion could be reached.

In this paper, we present a working system for translating stack-based Java virtual machine (JVM) code to a simple register code. We describe the translation process, the complicated parts of the JVM which make translation more difficult, and the optimisations needed to eliminate copy instructions. We also present a number of design choices, which can have a significant impact on the number of instructions in the resulting register machine program. We present quantitative results for real, large programs: the standard SPECjvm98 and Java Grande benchmark suites. We find that our virtual register architecture significantly reduces $(34.88 \%)$ the number of executed instructions in the Java programs we tested, although at the cost of increasing the number of bytecode fetches by $44.81 \%$. 
The rest of this paper is organised as follows. Section 2 describes the basic functioning of a virtual machine interpreter, and the most important types of instruction dispatch. In Section 3 we describe the main differences between virtual stack and virtual register machines. Section 4 looks at the particular strengths and weaknesses of the two types of virtual architecture, and estimates the relative advantages of each. In Section 5 we present our translation system for converting stack Java bytecode to an equivalent virtual register code. Section 6 examines techniques for eliminating move instructions from register code. Finally in Section 7 we present results showing that a register format can significantly reduce the number of executed instructions for the same program.

\section{Virtual machine interpreters}

The Java virtual machine uses a stack-based bytecode to represent the program. Interpreting a bytecode instruction consists of accessing arguments, performing the function of the instruction, and dispatching (fetching, decoding and starting) the next instruction.

Instruction dispatch typically consumes most of the execution time in virtual machine interpreters. The reason is that most VM instructions require only a small amount of computation, such as adding two numbers or loading a number onto the stack, and can be implemented in a few machine code instructions. In contrast, instruction dispatch can require up to 10-12 machine code instructions, and involves a time-consuming indirect branch. For this reason, dispatch consumes a the greater proportion of the running time of most efficient interpreters [7].

Switch dispatch is the simplest and most widely used approach. The main loop of the interpreter consists of a large switch statement with one case for each opcode in the JVM instruction set. Fig. 1 shows how this approach is implemented in C.

Switch dispatch is simple to implement, but rather inefficient for a number of reasons. First, most compilers produce a range check to ensure that the opcode is within the range of valid values. In the JVM this is unnecessary, since the bytecode verifier already checks that bytecodes are valid. Secondly, the break is translated into an unconditional jump back to the start of the loop. Given that the loop already contains a jump, it would be better to structure the loop as a set of routines that jump to one another. A final source of inefficiency results from there being only a single indirect branch for dispatching instructions. On machines with programmer visible pipelines, such as the Philips Trimedia processor for embedded systems, it is difficult to overlap this branch with other instructions [14]. On processors with current branch predictors, this branch is mispredicted more than $95 \%$ of the time [7].

An alternative to using a switch statement is threaded dispatch. Threaded dispatch is based on making explicit the sequence of steps generated by a compiler to implement a switch statement. Once these steps appear at the source level, the programmer can optimise the code by removing unnecessary work. Unfortunately, it is not possible to break a switch statement into its component parts in ANSI C, because there is no facility for goto statements that can jump to multiple different locations. To implement threaded dispatch, one requires a language with labels as first class value, such as GNU C, the language accepted by the GCC compiler. 


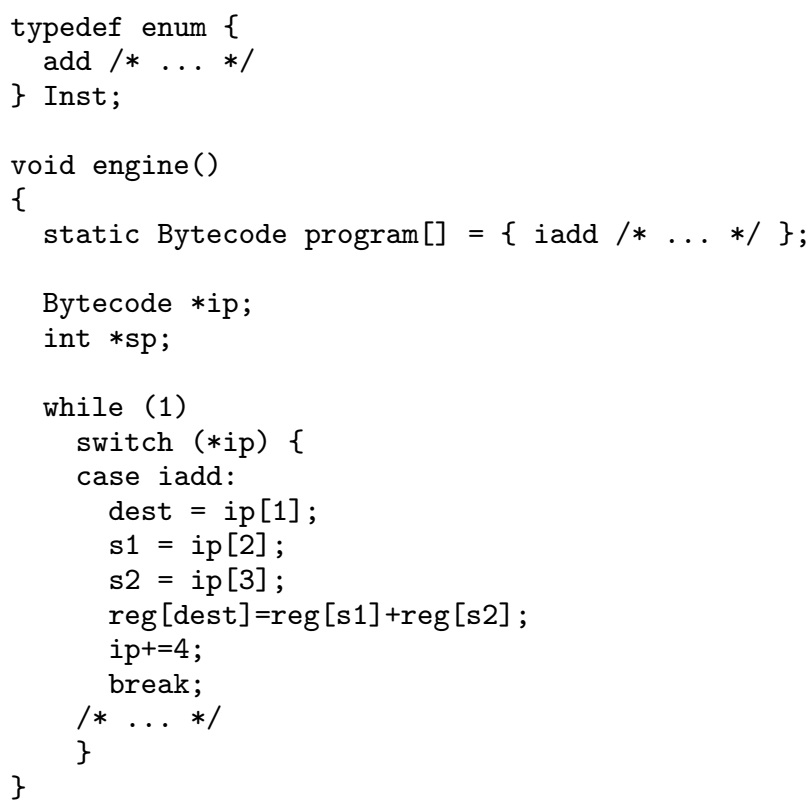

Fig. 1. Instruction dispatch using switch.

Fig. 2 shows how token threaded dispatch can be implemented using GNU C. The range check has been eliminated, as has the jump back to the dispatch routine at the end of the code for each VM instruction. Instead, the dispatch code is appended to the end of the code for each virtual machine instruction. This increases the size of the interpreter slightly, although it is usually faster. Another effect of replicating the dispatch code is that it allows the dispatch branch to be scheduled more efficiently with the code to implement the bytecode instruction, and it also greatly increases the prediction accuracy of the indirect branch on processors with branch target buffers (45\% versus $2 \%-20 \%$ for switch dispatch) [7].

If one is willing to build a more complicated interpreter system, even more efficient dispatch mechanisms can be used. For example, direct threaded dispatch [1] removes the cost of the table lookup by translating the bytecode to a format where each VM instruction is represented by a pointer to the $\mathrm{C}$ code to implement that instruction. This requires a pre-translation process, and greatly increases the size of the VM code (typically by a factor of 2-4 on a 32 bit machine).

Similarly, a variety of schemes have been proposed to reduce the cost and/or number of dispatches required to execute virtual machine code [8]. Essentially there are two main approaches. The first is to replicate the executable code to implement VM instructions, which improves the predictability of the indirect branch in the dispatch code. A second approach is to combine sequences of VM instructions into single "superinstructions" which perform the work of the full sequence, but require only a single dispatch to execute. Either 


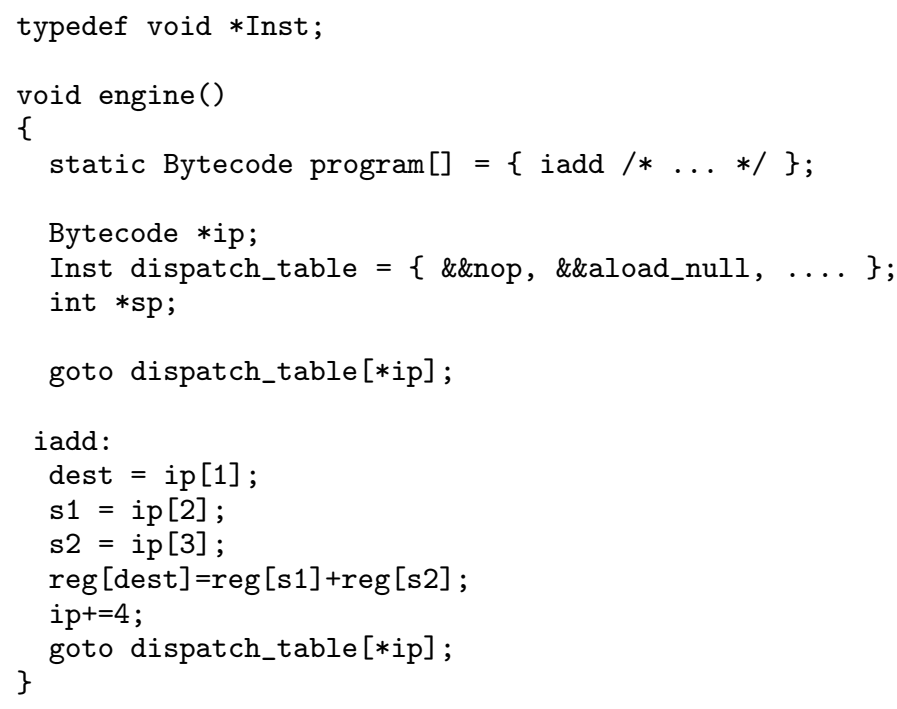

Fig. 2. Instruction dispatch using token threading in GNU C.

of these schemes can be implemented to work at either interpreter-build-time, or at the time when the interpreter is already running, with varying trade-offs in simplicity, code size and portability.

\section{Stack versus registers}

The cost of executing a virtual machine instruction consists of three components:

- Instruction dispatch.

- Operand access.

- Performing the computation.

The cost of dispatching an instruction is essentially the same for virtual register and stack machines. However, a given computation can often be expressed using fewer register machine instructions than stack ones. For example, the local variable assignment $\mathrm{a}=\mathrm{b}+\mathrm{c}$ might be translated to JVM code as ILOAD $\mathrm{c}$, ILOAD b, IADD, ISTORE a. In a virtual register machine, the same code would be the single instruction IADD a, b, c. Thus, virtual register machines have the potential to significantly reduce the number of executed instructions. By how much? It depends on how often values must be loaded to, stored from, or shuffled around the stack. If the computation can be organised so that operands can always be found on top of the stack, changing to a register architecture will give no reduction in executed instructions.

Another reason for pessimism with the number of executed instructions on register machines relates to register allocation. The number of virtual registers is always limited, 
and if there are more live values than registers, some values must be spilled to memory. Additional load and store instructions must be added to access spilled values, increasing the number of executed instructions rather than reducing them.

Our experience is that this argument is something of a red herring, at least for the Java VM. The most commonly used instructions for loading and storing local variables use a one-byte index, which specifies the number of the local variable. A comparable virtual register machine would use a one-byte index to specify each of its operands, allowing up to 256 virtual registers to be used in each method. Measurements show that no methods in the standard SPECjvm98 and Java Grande benchmarks contain anything like 256 local variables or stack values, the values that could be allocated to registers. On the contrary, most methods contain less than 25 such values [33]. ${ }^{1}$

The second component of the cost of executing a VM instruction is accessing the operands. This consists of two separate costs - finding the location of the operands, and accessing the operands themselves. Finding the operands' locations is expensive for a virtual register machine. The location of each operand must be fetched from the instruction stream, ${ }^{2}$ and used as an index into an array of virtual registers. In contrast the cost of locating operands is lower on a stack machine, since most operands are found on the top of the stack. The main cost is updating the stack pointer, and even this is not always necessary.

Virtual registers and virtual stacks are usually implemented as arrays in memory, so the costs of accessing the operands themselves are similar for the two types of virtual architecture. Stack caching can be used, however, to reduce the cost of accessing virtual stacks by keeping the top one or two items in a register. For example, Ertl [5] found that keeping the topmost stack item in a register reduced memory traffic for stack items by about $50 \%$.

It is very difficult to keep virtual register items in real machine registers, because real machine registers cannot be accessed in an array-like manner, with an index. However, virtual register machine instructions fetch and operate on all their data in a single VM instruction. Thus, intermediate values are likely to be kept in real machine registers during the execution of the VM instruction. In contrast, a virtual stack machine might require more than one VM instruction to perform the operation. The intermediate values between these different instructions are likely to be written to the stack, resulting in real machine load and store operations. Thus, a similar effect to stack caching can be achieved by the virtual register machine.

\footnotetext{
${ }^{1}$ Support for those very rare methods that contain more than 256 local variables could be implemented by adding special instructions move values in and out of a larger (16 bit indexed) local variable area. A few registers would be needed to load and store these 'spilled' values. In principle, a register allocator could be used to allocate the most important variables to the first 256 registers. However, such methods are likely to be so rare that the additional benefit of such an allocator would be so low as to make it unnecessary.

2 An alternative to fetching each operand location separately is to use a four-byte instruction containing the opcode and three register indices. This entire instruction could be fetched in a single load. However, it would still be necessary to extract the opcode and register numbers inside the four-byte instruction. This would involve shifting and masking the loaded instruction. Clearly the cost of such operations varies from one processor to another (for example the Pentium 4 has no barrel shifter, so large shifts are expensive). But the cost is likely to be in the same ball-park as the cost of four single-byte loads.
} 
The final component of the cost of executing a VM instruction is actually performing the computation itself. Given that most VM instructions perform a simple computation, such as an add or a load, this is usually the smallest part of the cost of executing a VM instruction. Generally, the type of virtual machine will not make a difference to this cost. The basic computation has to be performed, regardless of the format of our intermediate representation. However, there are situations where a register architecture can allow more efficient code. In particular, exploiting common sub-expressions (or more generally, partially redundant expressions) is easier on a register machine that does not destroy its operands when using them, as a stack machine normally does.

An important question is how great the benefit from eliminating partially redundant expressions might be on a virtual register machine. We are unaware of any measures of this optimisation on Java programs. However, Bodik et al. [2] measured the effect of this optimisation on the standard SPECint $95 \mathrm{C}$ benchmarks, and found that complete partial redundancy elimination allowed only around 3\% of operations to be eliminated. Furthermore, one of the most common sources of common sub-expressions is in the shifting of array subscripts, something that is not visible at the Java VM level. Therefore, the virtual register machine's advantage from being able to eliminate partially redundant expressions is likely to be small.

\section{Some estimates}

Clearly, the difference in speed between a virtual stack machine and a corresponding register machine can depend on many factors, especially on modern out-of-order processors with branch prediction and caches that make performance difficult to predict. However, we believe that the difference between the two types of machine can be estimated by looking at four main factors. The running of a virtual register machine (VRM) might be compared to a virtual stack machine (VSM) as follows:

$$
T_{V R M} \approx T_{V S M}-\# \text { dispatches } \times T_{\text {dispatch }}+\# \text { fetches } \times T_{\text {fetch }}
$$

In other words, the running time of a program on a VRM will be approximately equal to the running time of the program on a corresponding stack machine, minus the reduction in dispatches times the cost of a dispatch, plus the increase in fetches of operand locations times the cost of each of those fetches.

Generally, we would expect the increase in fetches to be large, since most VM instructions need between one and thee extra immediate operands to specify register locations. The cost of each of these fetches is likely to be low, however, since each usually corresponds to just an additional load instruction.

The reduction in dispatches is much more difficult to estimate without looking at real programs. However the cost of dispatch can be measured with at least some degree of accuracy. Ertl and Gregg [7] found that virtual machine interpreters contain very large numbers of indirect branches (up to $13 \%$ of all executed real machine instructions). Furthermore, these branches are highly (60\%-97\%) unpredictable on current desktop and workstation processors. The cost of each indirect branch misprediction is very high, because it requires that the entire pipeline be drained, consuming 6-30 cycles, depending 
on the length of the pipeline. Thus, an interpreter using threaded dispatch (typical indirect branch misprediction rate $60 \%$ ) running on a processor with a branch misprediction penalty of 20 cycles would expect to lose an average of 12 cycles on branch mispredictions for each VM instruction executed. Also we would expect the other dispatch code to take a couple of additional cycles to execute, for a total average dispatch cost of perhaps 14 cycles. Using switch dispatch would result in many more (around 97\% misprediction rate) indirect branch mispredictions, and a few more cycles for the other dispatch code.

Ertl and Gregg [7] found that more than half of the execution time of many efficient interpreters is spent on indirect branch mispredictions. Almost anything that reduces the number of dispatches has the potential to significantly improve performance. Therefore, if register virtual machines allow the same computation to be performed using fewer VM instructions, they may be significantly faster.

However, as noted above, the cost of all dispatch mechanisms is not the same; threaded dispatch is about twice as fast as switch dispatch, although it cannot be implemented in ANSI C. Similarly, other interpreter optimisations which reduce the cost and/or number of dispatches will strongly affect the relative performance of stack and register architectures. Thus, register machines might prove more efficient where the interpreter must be written in ANSI C for maximum portability, while a stack architecture might have an edge where GNU C or assembly language is acceptable.

\section{From stack to register}

To compare the relative benefits of virtual stack and register machines, we constructed a system for translating stack-based Java bytecode to a similar register code. The JVM performs almost all its computations on the stack. Values must be loaded from memory before they can be operated upon. JVM instructions consist of a (usually) single-byte opcode, followed by zero or more one-byte operands. Operands typically specify the location of local variables to be loaded, immediate arguments for arithmetic instructions, offsets to the program counter for branches, and indexes into the constant pool for instructions that invoke methods, access fields of objects, or use large constants.

Our register machine instruction set was chosen to provide direct counterparts to the JVM instructions. Each register machine instruction consists of a (usually) single-byte opcode, followed by exactly the same single-byte operands as in the JVM stack code. However, an additional one-byte immediate operand is added to specify the source and destination register of each value read from or written to the stack. ${ }^{3}$ Thus, most arithmetic instructions consist of only a single-byte opcode in the JVM, but the corresponding register instructions require an opcode and three operands.

Our translation scheme is based on mapping local variables and stack locations to a single set of virtual registers. In the JVM, all local variables are numbered, and we translate

\footnotetext{
${ }^{3}$ An exception is with some JVM local load and store instructions such as ILOAD_0, in which the immediate operand is encoded into the opcode. We use only a single-register MOVE instruction, in which both the source and destination are specified as immediate operands. Given that we eliminate most MOVE instructions, we judged that the benefit of specialised versions for particular source or destination registers would be small.
} 


\begin{tabular}{lll}
\hline ILOAD 4 & IMOVE $r 10, r 4$ & $;$ load local 4 \\
ILOAD 5 & IMOVE r11, r5 & ; load local 5 \\
IADD & IADD r10, r10, r11 & ; integer add \\
ISTORE 6 & IMOVE r6, r10 & $;$ store TOS to local 6 \\
ILOAD 6 & IMOVE r10, r6 & $;$ load local 6 \\
IFEQ 7 & IFEQ r10, 7 & $;$ branch by 7 if TOS $==0$ \\
\end{tabular}

Fig. 3. Example of stack and corresponding register code.

local variable numbers directly to virtual register numbers (so local variable zero is mapped to register zero).

Mapping stack locations to virtual register numbers is a little more complicated. Each stack location is given a number, and those numbers start just after the position of the last local variable. Mapping stack locations to register numbers is much simplified by Java's strict stack discipline. It is not possible to write code that, for example, increases the number of items on the stack on each iteration of a loop, as can be done in Forth. At every point in the program, the height of the stack must be fixed, and can be determined by simple static analysis. At control flow join points the stack heights must be equal on the two incoming control flow edges. Thus, by tracking the value of the stack pointer at each point in the program, it is possible to map stack locations to register numbers.

Fig. 3 shows an example of Java stack bytecode and the corresponding register code. Note that in the register code, the first register operand is always the destination. We assume that there are ten local variable slots in this method ( $r 0 \ldots r 9$ ), so the stack pointer for the initially empty stack will point to 10 . Thus, when we translate an ILOAD instruction which copies the value in local variable 4 to the top of the stack, we translate this as an integer move (IMOVE) instruction from register $r 4$ to register $r 10$.

Similarly, we translate the IADD stack instruction to a register IADD instruction that takes the topmost item in the stack ( $r 11)$ and the second from top ( $r 10)$, adds the two and places the result in the new topmost stack item ( 110$)$, which will be one lower than the previous top of stack because IADD reduces the height of the stack by one.

Using this scheme, it is relatively easy to translate any sequence of stack Java bytecode to an equivalent register format. It is important to note that the resulting code will often contain unnecessary and redundant MOVE instructions. For example, the original stack code contains the sequence ISTORE 6, ILOAD 6, which stores the topmost stack item to local variable number 6 , and then reloads the value to the top of the stack. This type of sequence is actually extremely common in code produced by the javac compiler. Presuming that the value is stored to the local variable to allow it to remain live after the end of the basic block, it is not possible to express this in fewer instructions. In the corresponding register code, however, it is easy to remove many of these IMOVE instructions.

One type of stack instruction that needs special handling in the translation is method invocation instructions. Standard (stack) JVM invoke instructions take their parameters from the top of the stack. These $n$ topmost stack items become the first $n$ local variables of the invoked method. Thus, invoke instructions can consume several values, and they destroy these values in the process. 
In theory, this scheme allows extremely fast parameter passing, since making the topmost stack elements into the first local variables simply involves one assignment to the frame pointer. In practice, however, the parameters are rarely already on the stack, and most JVM invoke instructions are preceded by one or more load instructions. Furthermore, once the parameters have been passed to the invoked method, they are in local variables and must be loaded to the stack before they can be used.

The simplest way to translate the parameter passing mechanism to register format would be a completely literal translation, where the topmost registers of the caller become the first registers of the callee. In our first implementation we used this scheme, but found that it prevented us from removing a very large number of load instructions when translating to register format. The problem is that invoke instructions consume several values, each of which must be in a specific register, and so cannot be moved.

Our new scheme uses an alternative scheme where each invoke takes as an immediate argument a list of the registers that it takes as parameters. Although this increases the number of loads necessary to identify the location of operands, it allows us to eliminate the great majority of MOVE instructions in register code.

\section{Eliminating moves}

Translating the Java bytecode to register code does not automatically reduce the number of executed instructions. The translation process outlined in the previous section simply converts each instruction directly from a stack to a register format. To eliminate unnecessary MOVE instructions, we apply a copy propagation algorithm that rewires the source and destination registers of instructions to bypass MOVEs. Once the sources and destinations have been changed, many of the MOVE instructions become dead code and can be eliminated.

We implemented two copy propagation algorithms, the first operating only on basic blocks and the second operating on the entire Java method. The basic block algorithm is both simple and efficient, and allows copy operations to be bypassed within a basic block. It is important to note that most values that are loaded to the stack are used very soon afterward, so a basic block algorithm can be very effective.

One complication that normally arises with single-basic-block copy propagation is that it is difficult to eliminate dead copies, because it is not clear which values are still alive at the end of the basic block. In Java bytecode, the problem is very much easier, since most destinations of MOVE instructions are on the stack. We can easily identify when most values on the stack become dead because the stack pointer moves below them (anything above the stack pointer is dead). Furthermore, the standard idiom used by the javac compiler is that the stack should be empty at the start and end of each statement. Thus, in the great majority of cases, the stack is empty at the end of each basic block, and all the items on the stack are dead.

Our second copy propagation algorithm operates on an entire method at a time. It uses classic dataflow analysis to compute liveness sets and propagate copies across basic block boundaries. Thus, it is much slower and more complicated that our basic block algorithm, and is probably not suitable for using in a scheme that translates from stack code to register code at load time. However, when we compared the two algorithms we found that there is 
a difference of less than $1 \%$ in the results. Clearly, a simple, efficient basic block approach with liveness based on stack position is sufficient in most cases.

\section{Experimental evaluation}

Our basic hypothesis is that virtual register machines have the potential to be interpreted more efficiently than stack machines by reducing the number of executed instructions. To test this hypothesis, we implemented a system for translating Java bytecode to a corresponding register format, and measured the differences using the SPECjvm98 [31] and Java Grande [3] benchmarks. These benchmarks consist of several large programs with real data, which are intended to be representative of a wide range of Java applications.

Our translation system was built into CVM, a small implementation of the Java 2 Micro Edition (J2ME) standard. It supports the full JVM instruction set, as well as full systemlevel threads. We made a number of small additions to CVM to enable it to run the SPEC benchmarks and to allow us to safely compile it at a higher level of optimisation than the standard distribution. All quoted figures are for the basic block implementation of copy propagation and dead copy elimination, as we believe this to be the most practical scheme. The whole-method copy propagation gives only slightly (less than 1\%) better results.

The first time each method is invoked we translate it to register format. Thus, we present measurements only for methods that are executed at least once. It is important to note that we do not advocate run-time translation from stack to register format as the best or only way to use virtual register machines. Clearly, this is a possibility, perhaps even an attractive one. But our main intention in doing this work is to evaluate free-standing virtual register machines. Run-time translation is simply a mechanism we use to easily compare stack and register versions of the JVM.

\subsection{Instruction dispatches}

Table 1 and Fig. 4 show the breakdown of instructions after translation to register format, based on statically appearing code. Overall, an average of $34.11 \%$ of statically appearing instructions are MOVE instructions. $28.56 \%$ of total instructions are MOVEs that can be eliminated with copy propagation and dead code elimination.

Table 2 and Fig. 5 show the breakdown of dynamically executed instructions. Interestingly, $41.28 \%$ of dynamically executed instructions are MOVEs. Clearly, local loads and stores are not distributed evenly throughout the programs, and code with larger numbers of such instructions tends to be executed more frequently. An average of $34.88 \%$ of executed instructions were eliminated by translating to a register format. At more than one third of executed instructions, this is a very large number and strongly suggests that our virtual register machine could be interpreted more efficiently than the corresponding stack machine. This is especially likely to be true where the interpreter uses switch dispatch (see Section 2), such as where the interpreter must be written in ANSI C.

\subsection{Code size and bytecode loads}

One of the drawbacks of register code is that it is usually larger than corresponding stack code, because the locations of operands must appear explicitly in the code. Fig. 6 and the 
Table 1

The number of static instructions that can be potentially removed and the number that are actually removed, compared with the total number of instructions

\begin{tabular}{llrlrll}
\hline Benchmark & Instructions & Moves & $(\%)$ & Eliminated & $(\%)$ & Code growth $(\%)$ \\
\hline compress & 28,612 & 9,852 & 34.43 & 8,227 & 28.75 & 43.26 \\
jess & 38,537 & 13,557 & 35.18 & 11,392 & 29.56 & 41.03 \\
db & 29,365 & 10,167 & 34.62 & 8,434 & 28.72 & 42.38 \\
javac & 59,545 & 22,442 & 37.69 & 19,179 & 32.21 & 36.94 \\
mpegaudio & 58,823 & 18,126 & 30.81 & 16,135 & 27.43 & 53.84 \\
mtrt & 33,969 & 12,004 & 35.34 & 10,079 & 29.67 & 42.72 \\
jack & 44,709 & 15,027 & 33.61 & 12,737 & 28.49 & 45.94 \\
MolDyn & 31,873 & 10,465 & 32.83 & 8,811 & 27.64 & 45.80 \\
RayTracer & 20,999 & 7,006 & 33.36 & 5,739 & 27.33 & 43.90 \\
Euler & 28,003 & 9,684 & 34.58 & 8,178 & 29.20 & 46.28 \\
MonteCarlo & 23,442 & 8,010 & 34.17 & 6,477 & 27.63 & 42.76 \\
Search & 21,328 & 7,005 & 32.84 & 5,717 & 26.81 & 45.08 \\
Average & 34,636 & $11,838.85$ & 34.11 & 9,982 & 28.56 & 44.17 \\
\hline
\end{tabular}

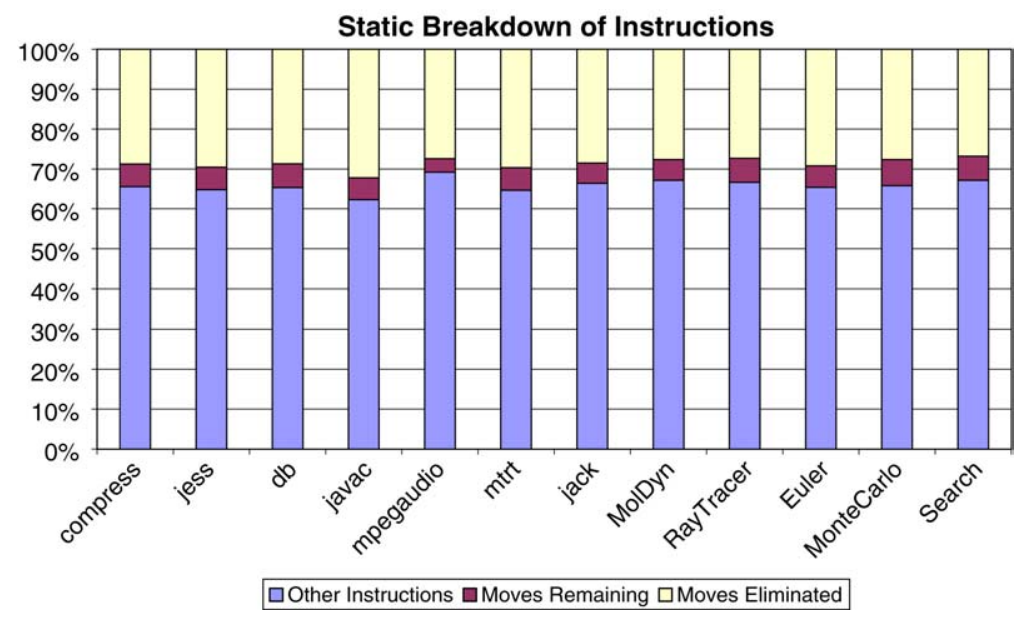

Fig. 4. Breakdown of statically appearing register code instructions into moves eliminated, moves that could not be eliminated and other instructions.

rightmost column of Table 1 shows the percentage increase in code size. There are two effects at work here, pulling in opposite directions. First, translating to a register format increases the number of operands in the bytecode. Secondly, applying copy propagation and dead copy elimination allows us to eliminate a large number of instructions, thus reducing both the number of opcodes and operands. The increases in code size are similar across all programs. Overall, the register code is an average of $44.17 \%$ larger than the corresponding stack code.

Perhaps the most important result of the increase in code size is that it will increase the number of real machine instructions required to load the bytecode instructions, including 
Table 2

The number of executed instructions (in millions) that can be potentially removed and that are actually removed, compared with the original total

\begin{tabular}{lrrlclll}
\hline Benchmark & Instructions & Moves & $(\%)$ & Eliminated & $(\%)$ & Extra loads & Loads/dispatch \\
\hline compress & 4,917 & 1,713 & 34.84 & 1372 & 27.91 & 5280 & 3.85 \\
jess & 979 & 419 & 42.82 & 349 & 35.73 & 657 & 1.88 \\
db & 1,135 & 541 & 47.68 & 428 & 37.69 & 750 & 1.75 \\
javac & 1,335 & 571 & 42.82 & 440 & 32.95 & 952 & 2.17 \\
mpegaudio & 4,805 & 1,779 & 37.04 & 1509 & 31.40 & 4387 & 2.91 \\
mtrt & 970 & 350 & 36.13 & 346 & 35.63 & 667 & 1.93 \\
jack & 611 & 277 & 45.40 & 229 & 37.48 & 347 & 1.52 \\
MolDyn & 7,589 & 3,663 & 48.26 & 3147 & 41.48 & 2883 & 0.92 \\
RayTracer & 7,177 & 2,654 & 36.98 & 2596 & 36.18 & 7594 & 2.92 \\
Euler & 10,162 & 4,100 & 40.35 & 3830 & 37.69 & 9082 & 2.37 \\
MonteCarlo & 1,625 & 609 & 37.52 & 498 & 30.67 & 1717 & 3.45 \\
Search & 4,780 & 1,924 & 40.26 & 1531 & 32.04 & 4563 & 2.98 \\
Average & 3,545 & 1,431 & 41.28 & 1252 & 34.88 & 2991 & 2.32 \\
\hline
\end{tabular}

The net increase in bytecode fetches (in millions) is shown in second rightmost column. The rightmost column shows the number of extra bytecode loads for each VM instruction dispatch eliminated.

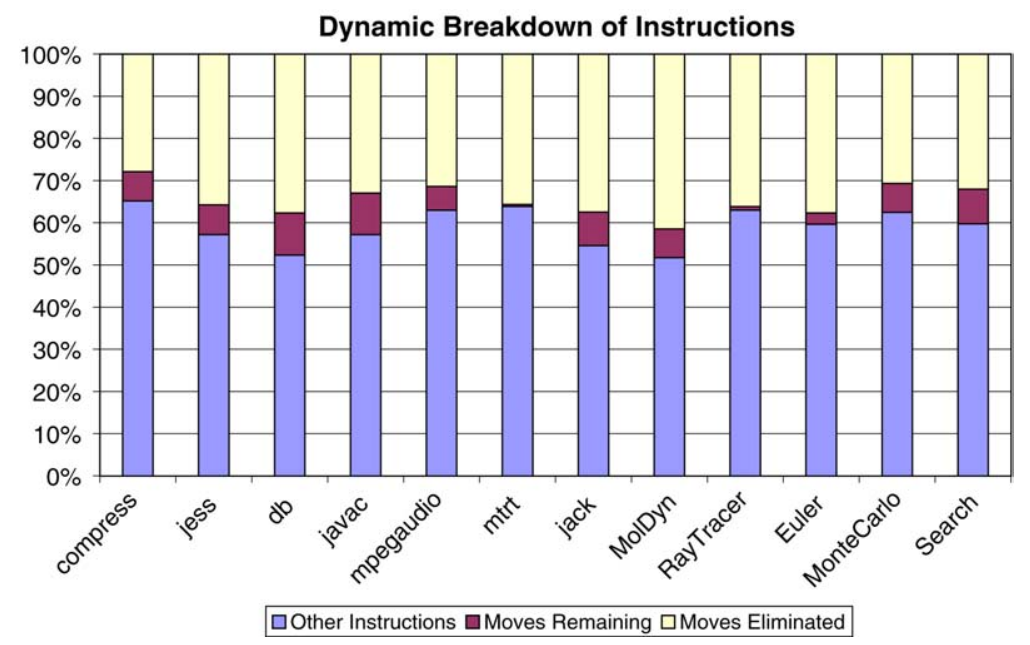

Fig. 5. Breakdown of dynamic register code instructions into moves eliminated, moves that could not be eliminated and other instructions.

operands. Fig. 6 shows the net increase in bytecode loads in the interpreter caused by the register format. These figures assume that each opcode and each register operand occupies one byte, each of which must be loaded separately. Whereas the code size increases are consistent across all programs, there is a wide variation in the number of additional dynamically executed loads caused by using a register architecture. This result is not necessarily surprising, because programs spend most of their time in small parts of the code, which may vary considerably from the rest of the program. Overall, the 


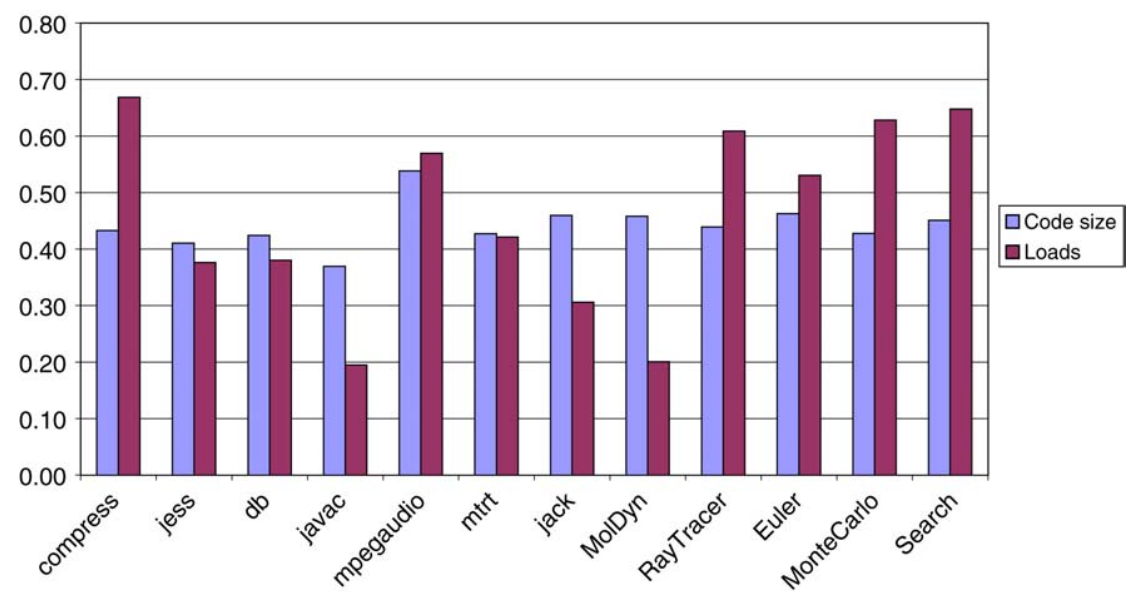

Fig. 6. Increase in code size and resulting net increase in bytecode loads from using a register rather than stack architecture.

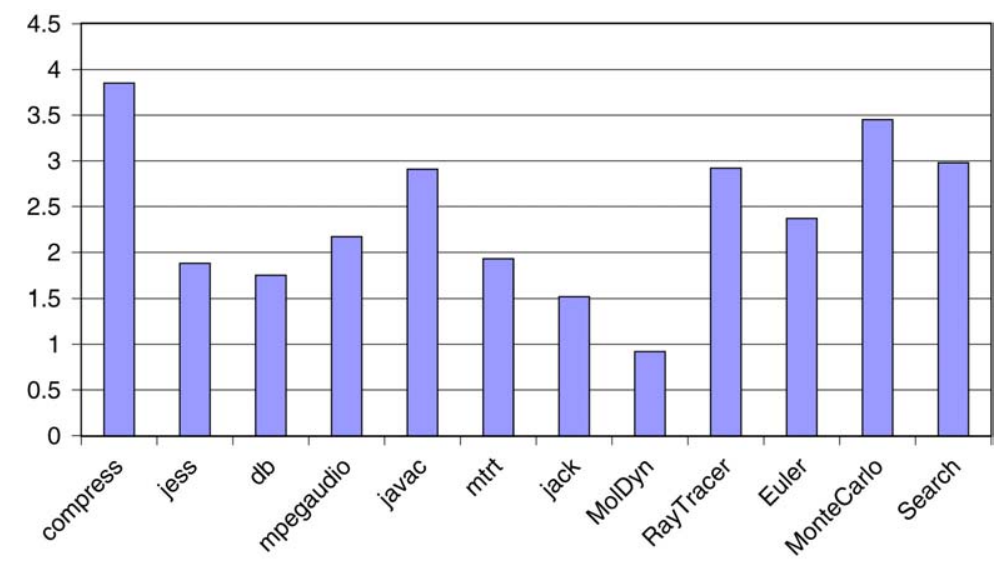

Fig. 7. Increases in dynamically loaded bytecode instructions per VM instruction dispatch eliminated by using a register rather than stack architecture.

register format requires an average of $44.81 \%$ extra bytecode loads. Clearly this is a large number, but loads are usually very much less costly than the indirect branches in instruction dispatches.

We also examined the ratio of the increase in the number of loads to the reduction in dispatches. That is, how many additional loads must be executed for each dispatch eliminated? As shown in Fig. 7 and the rightmost column of Table 2, there is a considerable variation from one program to another. For example, 0.92 extra loads per dispatch eliminated for MolDyn, compared with 3.85 for compress. As in Fig. 6, the number of dynamic bytecode loads is strongly influenced by relatively small pieces of frequently executed code. Overall for the SPECjvm98 and Grande benchmarks, translating to 


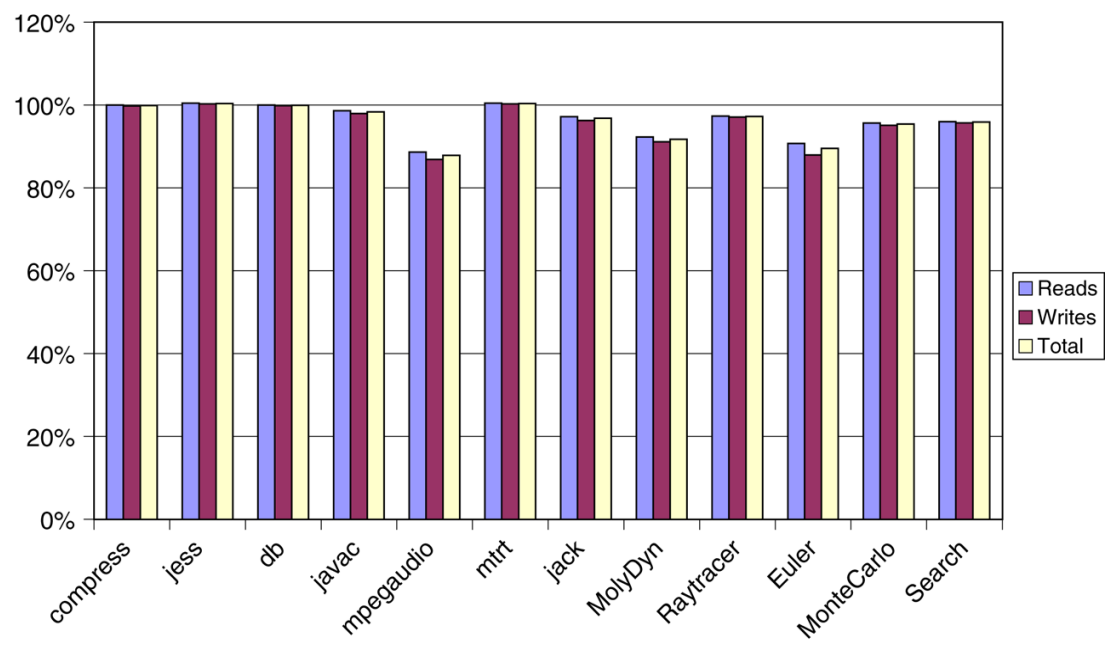

Fig. 8. Static number of real machine loads and stores required to access virtual registers in our virtual register machine as a percentage of the corresponding loads and stores for accessing the stack and local variables in a virtual stack machine.

bytecode increased the number of bytecode loads by an average of 2.32 for every dispatch eliminated. We believe that for switch-based interpreters running on modern pipelined processors where the cost of branch mispredictions is very high, even 2.32 extra loads for each dispatch removed will still result in a significant benefit to the virtual register machine interpreter.

\subsection{Local data memory accesses}

As was seen in Section 3, virtual register machine instructions fetch and operate on all their data in a single VM instruction. Thus, intermediate values are likely to be kept in real machine registers during the execution of the VM instruction. In contrast, a virtual stack machine must write these intermediate values to the stack. In many implementations, the stack is represented entirely as an array in memory, so these extra accesses correspond to real machine load and store instructions.

Fig. 8 shows the static number of loads and stores for accessing the virtual registers of our virtual register machine (assuming intermediate values within a VM instruction are kept in registers) as a percentage of the loads and stores needed to access the stack and local variables in the virtual stack machine. Overall, there is very little difference. Although there is some reduction in memory accesses due to intermediate operands being kept in registers, this is offset by our more clumsy parameter passing mechanism, which requires that all operands be copied.

In contrast, the dynamic reduction in memory accesses is much larger (Fig. 9). This is not surprising, given that more MOVEs are eliminated dynamically than statically (see Figs. 4, 5), and thus there is more potential for intermediate values to be kept in registers in the frequently executed code. 


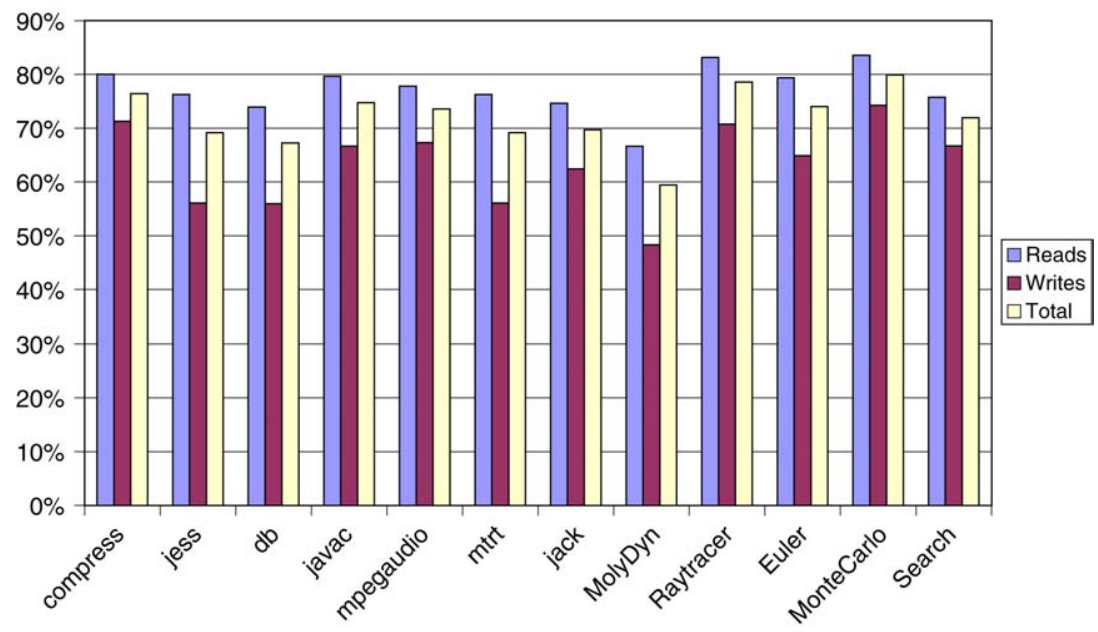

Fig. 9. Dynamic number of real machine loads and stores required to access virtual registers in our virtual register machine as a percentage of the corresponding loads and stores for accessing the stack and local variables in a virtual stack machine.

It is also interesting to note that the percentage reduction in stores is consistently greater than that in loads. Stores to the stack are mostly the result of JVM local load instructions such as ILOAD, which read a value from a local variable and write it to the stack. In contrast, a much smaller proportion of reads from the stack are the result of JVM local store instructions, such as ISTORE. Many other frequently executed VM instructions also read from the stack. So when the JVM code is translated to register code, and many of the MOVE instructions are eliminated, there is a disproportionate reduction in real machine stores. However, real machine loads are more frequent than real machine stores, so the total weighted reduction is closer to the reduction in real machine loads.

Fig. 10 shows the number of memory accesses eliminated per VM instruction executed. On average, 0.73 such memory accesses are eliminated per VM instruction executed, as compared with an average of 2.32 extra bytecode loads. So overall, there is still a significant increase in the number of real machine memory operations required when interpreting code for our register architecture.

Finally, it is important to note that these measurements assumes an unoptimised implementation of the virtual stack machine. The number of real machine loads and stores required for accessing values on the stack can be reduced dramatically using stack-caching [5]. So in optimised implementations, the stack architecture is likely to require fewer real machine loads and stores. Because these numbers depend so heavily on how the VM interpreter is implemented, we have not integrated them with our other measures of loads of instruction bytecodes.

In addition to the architecture-independent measurements presented in this paper, in the future we could like to present actual running times for stack and register versions of the JVM on various architectures. Although creating an interpreter for a register architecture is relatively simple, other aspects of the JVM such as concurrency, synchronisation, garbage 


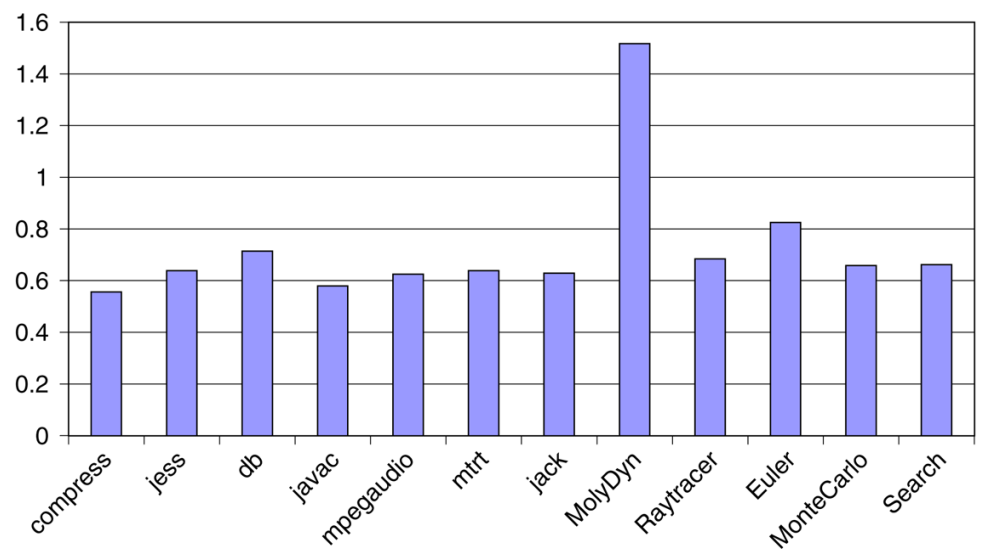

Fig. 10. Dynamic number of real machine memory accesses eliminated by converting to virtual register code per VM instruction executed.

collection, class verification, and exceptions make changing the layout of code and data in a JVM rather complicated. Initial experiments suggest that significant savings are indeed possible, but at the time of writing we have no properly working implementation.

\section{Related work}

Recent important developments in interpreters include the following. Interpreter generators simplify construction and maintenance of interpreters and can allow automatic VM instruction combining [27] and stack optimisations [9]. Stack caching [5] is a general technique for storing the topmost elements of the stack in registers. Ertl and Gregg [7] showed that interpreters (especially those using switch dispatch) spend most of their time in branch mispredictions on modern desktop architectures. Interpreter software pipelining [14] is a valuable technique for architectures with delayed branches (e.g. Philips Trimedia) or for preparing branch instructions (e.g. PowerPC), which makes the target of the dispatch branch available earlier by moving much of the dispatch code into the previous VM instruction. Costa [28] discusses various smaller optimisations.

The Sable VM [11] is an interpreter-based research JVM. This interpreter uses a runtime code generation system [25], not dissimilar from a just-in-time compiler. Sable uses a novel system of preparation sequences $[12,10]$ to deal with bytecode instructions that perform initialisations the first time they are executed. Such instructions otherwise make code generation difficult.

Myers [22] attempts to refute the idea that stack machines will necessarily result in smaller code, with lower cost to access operands. The argument is based on measurements of real programs which show that the expression in most assignment statements is extremely simple. Thus, in most cases operands must be loaded to the stack for use, rather than already being there as part of the evaluation of a complex expression. However, beyond measurements of the complexity of expressions, Myers presents only a handful of small examples showing situations where register code is superior to stack code. 
Myers' arguments led to a series of articles debating the topic in Computer Architecture News. Schulthess and Mumprecht [29] argue that Myers' measurements of the complexity of expressions are inconclusive, since programs contain features other than expressions that are better expressed using stacks. These include subroutine calls, parameter passing and multitasking. No quantitative data are provided.

Keedy [17] proposes an architecture using a both a stack and a single-register accumulator. He argues that the accumulator allows a number of common cases to be encoded in a smaller number of instructions than with the stack alone, without a large increase in code size. Myers [23] replies to this with further statistical data on expressions, arguing that two-address memory instruction sets can encode the commonest expressions most cheaply. Further discussions $[30,4,18]$ argue the merits of memory-to-memory and stack architectures.

The controversy between stack and register code has arisen again recently because of the decision to make the Parrot VM, the intermediate representation for the Perl 6 language, a register rather than stack machine. Again, arguments for this design decision [32] have been based on just a couple of small examples, rather than any study of real programs. The VM for the Lua [15] language also recently switched from a stack to a virtual machine, with the release of version 5.0. Similar suggestions were proposed by McGlasham and Bower [21] and Winterbottom and Pike [34], without studies of real programs.

\section{Conclusion}

Virtual register machines can be an attractive alternative to virtual stack architectures because they allow the number of executed instructions to be reduced by eliminating large number of loads to and stores from the stack. This is especially important for interpreters running on modern pipelined processors, where the cost of instruction dispatch is very high.

We have described a system for translating Java bytecode to a corresponding register format. We have implemented this system in a real JVM and used it to collect data on the effect of translating the SPECjvm98 and Java Grande benchmarks to register format. We believe that our study is the first to provide quantitative data from measuring hard numbers in real programs, rather than giving arguments based on small examples. We found that translating to a register format decreases the number of executed instructions by an average of $34.88 \%$, while increasing the number of bytecode loads by an average of $44.81 \%$. Overall, this corresponds to an increase of 2.32 loads for each dispatch removed. We believe that the high cost of dispatches makes register machines attractive even at the cost of increased loads.

\section{Acknowledgments}

We would like to thank the anonymous reviewers, whose comments greatly improved the quality of this paper. The reviewers of IVME 03 made a similar contribution to an earlier version of this paper. 


\section{References}

[1] J.R. Bell, Threaded code, Commun. ACM 16 (6) (1973) 370-372.

[2] R. Bodík, R. Gupta, M.L. Soffa, Complete removal of redundant expressions, in: PLDI'98 [26], 1998, pp. 1-14.

[3] M. Bull, L. Smith, M. Westhead, D. Henty, R. Davey, Benchmarking Java Grande applications, in: Second International Conference and Exhibition on the Practical Application of Java, Manchester, UK, April, 2000.

[4] R. Doran, Letter to the editor, Computer Architecture News 7 (1) (1978) 25-28.

[5] M.A. Ertl, Stack caching for interpreters, in: SIGPLAN'95 Conference on Programming Language Design and Implementation, 1995, pp. 315-327.

[6] M.A. Ertl, Implementation of stack-based languages on register machines, Ph.D. Thesis, Technische Universität Wien, Austria, 1996.

[7] M.A. Ertl, D. Gregg, The behaviour of efficient virtual machine interpreters on modern architectures, in: Euro-Par 2001, in: Springer LNCS, vol. 2150, 2001, pp. 403-412.

[8] M.A. Ertl, D. Gregg, Optimizing indirect branch prediction accuracy in virtual machine interpreters, in: Proceedings of the ACM SIGPLAN 2003 Conference on Programming Language Design and Implementation, PLDI 03, June 2003, San Diego, CA, ACM, 2003, pp. 278-288.

[9] M.A. Ertl, D. Gregg, A. Krall, B. Paysan, vmgen - A generator of efficient virtual machine interpreters, Software-Practice and Experience 32 (3) (2002) 265-294.

[10] E. Gagnon, A portable research framework for the execution of java bytecode, Ph.D. Thesis, McGill University, December 2002.

[11] E. Gagnon, L. Hendren, SableVM: A research framework for the efficient execution of Java bytecode, in: First USENIX Java Virtual Machine Research and Technology Symposium, Monterey, CA, April, 2001.

[12] E. Gagnon, L. Hendren, Effective inline-threaded interpretation of java bytecode using preparation sequences, in: Proceedings of the 12th International Conference on Compiler Construction, April 2003, in: LNCS, vol. 2622, 2003, pp. 170-184.

[13] D. Gregg, A. Ertl, A. Krall, A fast java interpreter, in: Proceedings of the Workshop on Java Optimisation Strategies for Embedded Systems, JOSES, Genoa, April, 2001.

[14] J. Hoogerbrugge, L. Augusteijn, J. Trum, R. van de Wiel, A code compression system based on pipelined interpreters, Software-Practice and Experience 29 (11) (1999) 1005-1023.

[15] R. Ierusalimschy, Programming in Lua, ISBN: 85-903798-1-7, 2003, http://www.Lua.org.

[16] A.C. Kay, The early history of smalltalk, in: History of Programming Languages, ACM Press/AddisonWesley, 1996, pp. 511-579.

[17] J.L. Keedy, On the use of stacks in the evaluation of expressions, Computer Architecture News 6 (6) (1978) $22-28$.

[18] J.L. Keedy, More on the use of stacks in the evaluation of expressions, Computer Architecture News 7 (8) (1979) 18-21.

[19] P.J. Koopman Jr., Stack Computers, Ellis Horwood Limited, 1989.

[20] G. Krasner (Ed.), Smalltalk-80: Bits of History, Words of Advice, Addison-Wesley, 1983.

[21] B. McGlashan, A. Bower, The interpreter is dead (slow). isn't it? in: OOPSLA'99 Workshop: Simplicity, Performance and Portability in Virtual Machine Design, 1999.

[22] G.J. Myers, The case against stack-oriented instruction sets, Computer Architecture News 6 (3) (1977) 7-10.

[23] G.J. Myers, The evaluation of expressions in a storage-to-storage architecture, Computer Architecture News 6 (9) (1978) 20-23.

[24] S. Pemberton, M. Daniels, Pascal Implementation — The P4 Compiler, Ellis Horwood, 1982.

[25] I. Piumarta, F. Riccardi, Optimizing direct threaded code by selective inlining, in: PLDI'98 [26], 1998, pp. 291-300.

[26] SIGPLAN'98 Conference on Programming Language Design and Implementation, 1998.

[27] T.A. Proebsting, Optimizing an ANSI C interpreter with superoperators, in: Principles of Programming Languages, POPL'95, 1995, pp. 322-332.

[28] V. Santos Costa, Optimising bytecode emulation for Prolog, in: Proceedings of PPDP'99, September 1999, in: LNCS, vol. 1702, Springer-Verlag, 1999, pp. 261-267.

[29] P. Schulthess, E. Mumprecht, Reply to the case against stack-oriented instruction sets, Computer Architecture News 6 (5) (1977) 24-27. 
[30] R. Sites, A combined register-stack architecture, Computer Architecture News 6 (8) (1978) 19.

[31] SPEC, SPEC releases SPEC JVM98, first industry-standard benchmark for measuring Java virtual machine performance, Press Release, August 19 1998. http://www.specbench.org/osg/jvm98/press.html.

[32] D. Sugalski, http://www.parrotcode.org/.

[33] J. Waldron, Dynamic bytecode usage by object oriented java programs, in: Proceedings of the Technology of Object-Oriented Languages and Systems 29th International Conference and Exhibition, 7-10 June 1999, Nancy, France.

[34] P. Winterbottom, R. Pike, The design of the Inferno virtual machine, in: IEEE Compcon 97 Proceedings, San Jose, CA, 1997, pp. 241-244. 